\title{
Comportamiento predestete de cabritos cruzados en Guanajuato, México
}

\author{
Pre-weaning performance of crossbred goat kids from Guanajuato, \\ Mexico
}

\author{
Sonia Castillo-Rodríguez, ${ }^{1}$ M.Sc, Jorge Rivera-Sandoval, ${ }^{2}$ M.Sc, \\ Arnoldo González-Reyna, ${ }^{1}$ Ph.D, Juan Martínez-González, ${ }^{* 1}$ Ph.D.
}

${ }^{1}$ Universidad Autónoma de Tamaulipas, Facultad de Ingeniería y Ciencias, Secretaría de Posgrado e Investigación. Tamaulipas, México. ${ }^{2}$ Gobierno del Estado de Guanajuato. Guanajuato, México. *Correspondencia: jmartinez@uat.edu.mx

Recibido: Abril de 2012; Aceptado: Mayo de 2013.

\section{RESUMEN}

Objetivo. Los pesos al nacimiento y al destete fueron registrados para evaluar el comportamiento predestete de cabritos cruzado en ocho pequeñas fincas de producción de leche de cabra en Guanajuato, México. Materiales y métodos. Se usaron 234 registros de cabritos, las cabras fueron mantenidas en condiciones de pastoreo con suplementación de un concentrado comercial. Los cabritos eran cruzados, de las razas Sannen, Alpina y Toggenburg, los datos fueron analizados con un modelo estadístico que considero los efectos de rebaño (RE), grupo contemporáneo (GC), número de parto (NP), tipo parto (TP) y sexo del cabrito (SC). Resultados. Las medias de peso al nacer, peso a los 60 días de edad y la ganancia diaria de peso fueron $3.4 \pm 0.5$ (rango 2.0 a 5.5), $13.7 \pm 1.8$ (rango 8.0 a 17.5 ) y $0.17 \pm 0.02$ $(0.08$ a $0.23 \mathrm{~kg})$. El peso al nacimiento fue afectado $(\mathrm{p}<0.01)$ por RE, GC, NP, TP y SC. Sin embargo, el peso a 60 días y ganancia diaria de peso fueron afectadas $(p<0.05)$ por RE y SC. Conclusiones. El rebaño afectó el comportamiento de los cabritos.

Palabras clave: Cabritos, comportamiento, rebaños (Fuente: CAB).

\section{ABSTRACT}

Objective. Birth and weaning weights were recorded, to valuate pre-weaning performance of crossbred kids, in eighth small milk goat flocks, from Guanajuato, Mexico. Materials and methods. Records of 234 kid dings were used; the goats were kept under grazing rangeland with supplementation of commercial concentrate. The kids were crossbred between Sannen, Alpine and Toggenburg breeds. Data was analyzed by a statistical model in which the flock effects (FL), contemporary group (CG), parity number (PN), litter size (LS) and sex of the kid (SK) were considered. Results. Birth weights averaged $3.4 \pm 0.5 \mathrm{~kg}$ (range 2 to $5.5 \mathrm{~kg}$ ) and weight at 60 days of age averaged $13.7 \pm 1.8 \mathrm{~kg}$ (range 8.0 to $17.5 \mathrm{~kg}$ ); and daily weight gain averaged $0.17 \pm 0.02 \mathrm{~kg}$ (range 0.08 to $0.23 \mathrm{~kg}$ ). Birth weights were affected $(p<0.01)$ by FL, CG, PN, LS and SK. However, weight at 60 days and daily weight gain were affected $(p<0.05)$ by FL and SK. Conclusions. The flock affected the performance of the kids.

Key words: Flocks, goats kids, performance (Source: $C A B$ ). 


\section{INTRODUCCIÓN}

En México el aprovechamiento de la cabra como animal productor de carne y leche data desde la época de la colonia, así fue como se generalizó el consumo de su carne a través de platillos como: el cabrito al pastor, la birria y la barbacoa. Hoy en día la caprinocultura se ha vuelto una actividad ganadera atractiva para los productores de bajos recursos (1). Es bien conocida la capacidad que tienen las cabras para convertir los forrajes toscos en fuentes de alimentos como la carne y la leche. También, las cabras proporcionan una diversidad de productos, destacando su fibra que se usa para la elaboración de mohair y vestidos de casimir.

El inventario de ganado caprino para el Estado de Guanajuato en el año de 2008 fue de 559,239 cabezas (2), en aproximadamente 400 granjas que se dedican a la producción de leche. El tamaño del rebaño en las granjas puede estar constituido desde 10 cabezas hasta algunos cientos de cabras con un promedio de 70 cabezas (3).

Algunos factores que limitan la productividad de las cabras son: el sistema de producción, los programas de alimentación, falta de programas reproductivos y falta de organización para la comercialización de los productos.

En México se pueden caracterizar tres sistemas de producción: el primero lo constituyen los sistemas extensivos, que se basan en el aprovechamiento de los agostaderos de las zonas áridas y semiáridas y en los que predominan los fenotipos Criollos o encastados con nubiana; el principal producto es el cabrito mamón y los animales de desecho. El segundo grupo lo conforman los sistemas semi-intensivos, que se ubican principalmente en la Laguna y el Bajío, generalmente manejan rebaños encastados o de razas especializadas como Saanen y Alpina, la base de la alimentación la conforman el pastoreo, el aprovechamiento de esquilmos agrícolas y concentrados. Mientras que el tercer grupo está conformado por sistemas intensivos (estabulados), dedicados a la producción de leche y/o de pie de cría.

Por otro lado, el mejoramiento genético de los caprinos deberá contestar algunas interrogantes para decidir el plan a seguir, como son: cuál es el sistema de producción, con qué razas, con qué recursos y las demandas del mercado al que se quiere acceder. Es importante mencionar que los pesos y las ganancias diarias de peso en cabritos se han mejorado. Sin embargo, no se conocen en México resultados en sistemas de producción semi-intensivos, como es el caso del altiplano.
Por tanto, el objetivo de este trabajo fue evaluar los efectos de algunos factores ambientales (rebaño, grupo contemporáneo, número de parto, tamaño de la camada y sexo del cabrito) sobre comportamiento productivo (peso al nacimiento, peso al destete y ganancia diaria de peso) de cabritos en el Estado de Guanajuato, México.

\section{MATERIALES Y MÉTODOS}

Sitio de estudio. El presente trabajo se realizó con la información registrada en ocho fincas caprinas del Estado de Guanajuato, México. Localizado geográficamente a los $100^{\circ} 59^{\prime} 50^{\prime \prime}$ LO, $20^{\circ} 37^{\prime} 35^{\prime \prime} \mathrm{LN}$ y a $1.750 \mathrm{msnm}$. El clima está clasificado como semi-caluroso y subalternohúmedo con las Iluvias en verano, la precipitación y temperatura media anual son de $628 \mathrm{~mm}$ y $19.5^{\circ} \mathrm{C}$, respectivamente (3).

La vegetación está principalmente compuesta por bosques de encino (Querqus spp.), matorral espinoso (Acacias spp.) y pastizal amacollado arborescente, con predominio de especies forrajeras como zacatón (Panicum máximum Jacq.), lobero (Buchloe dactyloides Nutt. Engelm), navajita (Bouteloua gracilis H.B.K. Lag. ex Steud), colorado (Panicum coloratum L.), lanudo (Holcus lanatus), cola de zorra (Setaria parvifolia), banderita (Bouteloua curtipendula Michx. Torr.), búfalo (Trichoposgon capitatum), mezquite (Prosopis laevigata Humb. \& Bonpl. ex Willd. M. C. Johnst.) y otras especies como pingüica (Arctostaphylos pungens), nopal (Opuntia spp.), largoncillo (Acacia constricta var. Vernicosa standl), tepehuajes (Acacia acapulsensis Kunth.), palo blanco (Calycophyllun multiflorum), órgano (Carnegea gigantea) y garambullo (Myrtillocactus geometrizans) (4).

Muestra. Entre las fincas existe una gran diversidad en cuanto a infraestructura, algunas cuentan solo con corrales rústicos para el confinamiento del rebaño, construidos con postes de madera, tela borreguera y dos hilos de alambre de púas en la parte superior. Mientras que otras cuentan con corrales de mampostería y equipados para alojar un gran número de animales.

La alimentación consistió en el pastoreo de praderas nativas e introducidas y suplementación con un concentrado comercial, la reproducción fue con inseminación artificial y monta directa.

Al momento del parto los cabritos fueron pesados en las primeras 24 horas de vida y la cría fue natural para ser destetados aproximadamente 
a los 60 días de edad, por lo que los pesos al destete fueron ajustados a esta edad. La ganancia de peso diaria fue estimada (GPD) por la diferencia entre pesos del nacimiento al destete divido entre los días trascurridos.

Análisis estadístico. Los datos fueron analizados usando el procedimiento de modelos lineales generales (5) que consideró los efectos de finca, grupo contemporáneo (GC), número de parto, tipo de parto, sexo del cabrito y sus interacciones, mediante el siguiente modelo estadístico:

$\mathrm{Y}_{\mathrm{ijk} k \mathrm{~m}}=\mu+\mathrm{FI}_{\mathrm{i}}+\mathrm{GC}_{\mathrm{j}}+\mathrm{NP}_{\mathrm{k}}+\mathrm{TP}_{\mathrm{l}}+(\mathrm{NP} * \mathrm{TP})+\mathrm{SC}_{\mathrm{m}}+\mathrm{E}_{\mathrm{ijk} k \mathrm{~m}}$

Donde:

$\mathrm{Y}_{\mathrm{ijklm}}=$ variable de respuesta (peso al nacimiento, peso al destete y ganancia diaria de peso)

$\mu=$ media general

$\mathrm{FI}_{\mathrm{i}}=$ efecto de la i-ésima finca,

$\mathrm{GC}_{\mathrm{j}}=$ efecto del j-ésimo grupo contemporáneo,

$\mathrm{NP}_{\mathrm{k}}=$ efecto del k-ésimo número de parto,

$\mathrm{TP}_{1}=$ efecto del l-ésimo tipo de parto,

(NP*TP) interacción,

$\mathrm{SC}_{\mathrm{m}}=$ efecto del m-ésimo sexo del cabrito y

$\mathrm{E}_{\mathrm{ijk} \mid m n}=$ error experimental $\sim\left(0, \sigma^{2}\right)$.

Las diferencias entre medias de los efectos principales fueron estimadas a través de la prueba de Duncan (5).

\section{RESULTADOS}

En el presente estudio la media general para peso al nacimiento fue de $3.41 \pm 0.83 \mathrm{~kg}$. En la tabla 1 se puede observar que existieron diferencias significativas $(p<0.01)$ de finca $y$ grupo contemporáneo (GC). Los cabritos del GC 1 pesaron $1.07 \mathrm{~kg}$ más al nacimiento que los del GC 5 (Tabla 2). Los pesos de los cabritos variaron entre las fincas, siendo más pesados en las explotaciones donde se daban los niveles más altos de suplementación.

Tabla 1. Análisis de varianza para peso al nacer (PN), peso al destete (PD) y ganancia diaria de peso predestete (GDP) de cabritos en Guanajuato, México.

\begin{tabular}{|c|c|c|c|c|c|c|c|c|c|}
\hline \multirow{2}{*}{$\begin{array}{l}\text { Fuente de } \\
\text { variación }\end{array}$} & \multicolumn{3}{|c|}{ PN } & \multicolumn{3}{|c|}{ PD } & \multicolumn{3}{|c|}{ GDP } \\
\hline & gl & CM & $\mathrm{Pr}>\mathrm{F}$ & gl & CM & $\operatorname{Pr}>\mathrm{F}$ & $\mathrm{gl}$ & CM & $\mathrm{Pr}>\mathrm{F}$ \\
\hline $\begin{array}{l}\text { Finca } \\
\end{array}$ & 7 & 2.63 & ** & 4 & 5.06 & * & 4 & 0.001 & * \\
\hline $\begin{array}{l}\text { Grupo } \\
\text { contemporáneo }\end{array}$ & 4 & 2.55 & ** & 4 & 3.20 & NS & 4 & 0.001 & NS \\
\hline $\begin{array}{l}\text { Número de } \\
\text { parto }\end{array}$ & 4 & 2.02 & ** & 4 & 2.59 & NS & 4 & 0.001 & NS \\
\hline $\begin{array}{l}\text { Tamaño de la } \\
\text { camada }\end{array}$ & 2 & 4.76 & $* *$ & 2 & 0.20 & NS & 2 & 0.001 & NS \\
\hline Sexo de la cría & 1 & 12.67 & ** & 1 & 9.66 & * & 1 & 0.003 & * \\
\hline NP*TC & 6 & 1.20 & ** & 5 & 0.91 & NS & 5 & 0.003 & NS \\
\hline Peso al nacer & & & & 1 & 78.59 & ** & 1 & 0.002 & NS \\
\hline Error & 209 & 0.35 & & 121 & 1.58 & & 121 & 0.001 & \\
\hline Total & 233 & & & 142 & & & 142 & & \\
\hline
\end{tabular}

$\mathrm{gl}=$ grados de libertad, $\mathrm{CM}=$ Cuadrado medio, $* *=\mathrm{p}<0.01, *=\mathrm{p}<0.05$, NS=no significativo.
Tabla 2. Medias de peso al nacer (PN), peso al destete (PD) y ganancia diaria de peso predestete (GDP) de cabritos en Guanajuato, México.

\begin{tabular}{ccccccc}
\hline Característica & \multicolumn{2}{c}{ PN (kg) } & \multicolumn{2}{c}{ PD (kg) } & \multicolumn{2}{c}{ GDP (g) } \\
& $\mathbf{n}$ & Media & $\mathbf{n}$ & Media & $\mathbf{n}$ & Media \\
\hline Grupo contemporáneo & & & & & & \\
1 & 42 & $4.16^{\mathrm{a}}$ & 29 & 14.50 & 29 & 166 \\
2 & 19 & $3.32^{\mathrm{b}}$ & 19 & 13.21 & 19 & 165 \\
3 & 39 & $3.31^{\mathrm{b}}$ & 33 & 13.23 & 33 & 166 \\
4 & 100 & $3.27^{\mathrm{b}}$ & 39 & 13.64 & 39 & 170 \\
5 & 34 & $3.04^{\mathrm{b}}$ & 23 & 13.59 & 23 & 173 \\
Tamaño de la camada & & & & & & \\
Sencillo & 45 & $3.62^{\mathrm{a}}$ & 35 & 13.74 & 35 & 168 \\
$\begin{array}{c}\text { Gemelos } \\
\text { Triates }\end{array}$ & 152 & $3.43^{\mathrm{a}}$ & 91 & 13.67 & 91 & 168 \\
Sexo del cabrito & $3.04^{\mathrm{b}}$ & 17 & 13.41 & 17 & 166 \\
Macho & 114 & $3.67^{\mathrm{a}}$ & 69 & $14.31^{\mathrm{a}}$ & 69 & 174 \\
Hembra & 120 & $3.16^{\mathrm{b}}$ & 74 & $13.04{ }^{\mathrm{b}}$ & 74 & $162^{\mathrm{b}}$ \\
\hline
\end{tabular}

$a, b, c$ son diferentes $p<0.05$

De igual modo, se observaron diferencias significativas $(p<0.01)$ de número y tipo de parto (Tabla 1). En este estudio las cabras de cinco partos produjeron los cabritos más pesados al nacimiento (3.81 kg; Figura 1). En cuanto al sexo del cabrito este también tuvo efectos significativos $(p<0.01)$ sobre el peso al nacer (Tabla 1). Los cabritos pesaron $0.50 \mathrm{~kg}$ más al nacimiento que las hembras (Tabla 2 ).

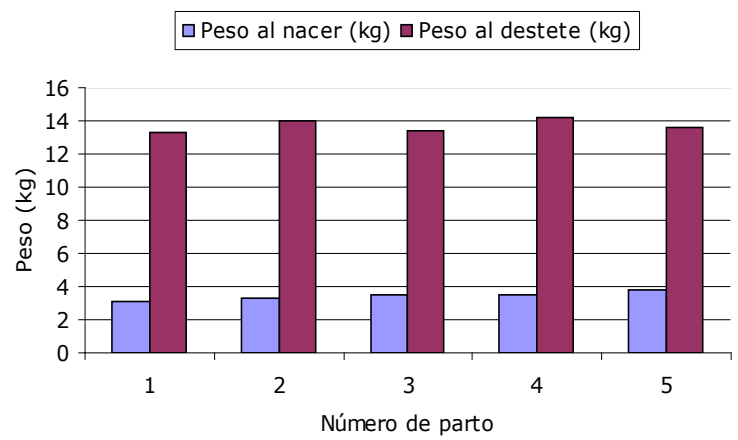

Figura 1. Medias de peso al nacer y peso al destete de cabritos de razas lecheras de acuerdo al número de parto de la cabra en Guanajuato, México.

Con relación al peso al destete ajustado a 60 días se encontró una media de $13.65 \pm 1.79$ $\mathrm{kg}$. En este estudio solo la finca y el sexo de la cria afectaron significativamente $(p<0.01)$ los pesos al destete (Tabla 1). Los cabritos machos superaron a las hembras en $1.27 \mathrm{~kg}$ al destete (Tabla 2). El peso al nacimiento (como covariable) afectó el peso al destete $(p<0.01)$, los cabritos más pesados al nacimiento también fueron más pesados al destete.

Con relación a la ganancia diaria de peso la media fue de $168 \pm 24 \mathrm{~g}$. En la tabla 1 se puede apreciar que la finca y el sexo del cabrito afectaron $(p<0.01)$ la ganancia diaria de peso. En este estudio los machos superaron a las hembras en $12 \mathrm{~g}$ más la ganancia diaria de peso (Tabla 2). 


\section{DISCUSIÓN}

En el presente estudio la media de peso al nacimiento fue similar a la citada por Peña et al (6), Martínez et al (7), Merlos et al (8) y Leal et al (9), Mellado et al (10) y Martínez et al (11) quienes encontraron que el peso al nacer de los cabritos alcanzó los $3.4 \mathrm{~kg}$ en promedio. Sin embargo, Martínez et al (12) encontraron pesos inferiores al del presente estudio. Brown et al (13) reportaron que los cabritos hijos de sementales Boer alcanzaron pesos al nacer de $4.1 \mathrm{~kg}$ por tratarse de una raza netamente productora de carne.

Como era de esperarse la finca y el grupo contemporáneo afectaron los pesos de los cabritos, siendo más pesados en las explotaciones donde se daban los niveles más altos de suplementación. Merlos et al (8) también encontraron efecto significativo del año de nacimiento sobre el peso al nacer. Resultados que pueden ser atribuidos a las diferencias ambientales entre años en cuanto a precipitación, dado que la disponibilidad de forraje esta íntimamente relacionada con esta. Sin embargo, Martínez et al (7) no observaron efecto del mes de nacimiento en rebaños bajo condiciones similares de manejo.

De igual modo, el número y tipo de parto fueron fuentes importantes de variación en el peso al nacer de los cabritos. Martínez et al (12) encontraron que los pesos al nacer para cabritos de simples, mellizos y trillizos fueron de $3.4,3.0$ y $2.6 \mathrm{~kg}$, respectivamente. Similarmente, Merlos et al (8) citaron que los pesos al nacer de cabritos fueron 3.5 y $3.1 \mathrm{~kg}$ para los sencillos y gemelares, respectivamente. En este estudio los cabritos machos pesaron más al nacimiento $(8,12)$.

El peso al destete fue similar al de otros estudios $(8,9)$. Sin embargo, Martínez et al $(12)$ encontraron un peso inferior (13.2 kg) para cabritos destetados en fincas ubicadas en la misma área de estudio.
Al igual que en el peso al nacimiento la finca y el sexo del cabrito afectaron el peso al destete. Merlos et al (8) encontraron que el sexo del cabrito afectaba el peso al destete en el trópico seco del Estado de Guerrero. Las diferencias pueden probablemente ser explicadas por el manejo entre las fincas y los niveles de suplementación que suministraban individualmente. Además, en casi todas las especies, los machos siempre son más pesados que las hembras.

La media de ganancia diaria de peso encontrada en este estudio se puede considerar como aceptable, otros investigadores $(14,15)$ encontraron ganancias diarias de peso a las 10 semanas de edad de $109 \mathrm{~g}$. Similarmente, Díaz et al (16) encontraron que la ganancia diaria de peso de cabritos alimentados con diferentes niveles de soya en el sustituto de leche, tuvieron ganancias diarias de $138 \mathrm{~g}$.

En este estudio la finca y el sexo del cabrito fueron fuentes de variación importantes en la ganancia diaria de peso. Meneses et al (14) al trabajar con diferentes manejos de la alimentación encontraron efectos significativos debido a manejo $(p<0.05)$. El diferente comportamiento de los cabritos entre fincas se puede explicar probablemente por las diferencias en manejo y nivel de suplementación.

En conclusión los datos presentados aquí, representan una muestra muy pequeña de la población de cabras del Estado de Guanajuato, México por lo que los resultados deben interpretarse como tal. El peso al nacimiento, la ganancia diaria de peso y el peso al destete fueron más elevados que los tradicionalmente esperados para cabras productoras de leche. Los efectos de la finca sobre el comportamiento de los cabritos dan indicios que se pueden mejorar los parámetros productivos si se homogenizan los manejos entre las fincas. Como en casi todas las especies el número de parto de la cabra afectó el peso al nacer de las crías.

\section{REFERENCIAS}

1. Hernández ZJ. La caprinocultura en el marco de la ganadería poblana (México): Contribución de la especie caprina y sistemas de producción. Arch Zootec 2000; 49: 341-352.
2. Sistema de Información Agroalimentaria y Pesquera. 2008 (fecha de acceso 15 de marzo de 2010). México DF. Disponible en http://www.siap.gob.mx/ventana. php?idLiga $=992 \&$ tipo $=1$ 
3. Instituto Nacional de Estadística Geografía e Informática. 2007 (fecha de acceso 15 de octubre de 2009). México DF Disponible en http://www.inegi.gob.mx/inegi/default. asp $\mathrm{x} ? \mathrm{~s}=\mathrm{geo} \& \mathrm{c}=\& \mathrm{e}=11$

4. Carranza GE. Conocimiento actual de la flora y la diversidad vegetal del Estado de Guanajuato, México. Primera Semana Estatal de Ecología Guanajuato; 2002 Jun 3 a 7; Guanajuato, Guanajuato, México. Instituto de Ecología del Estado de Guanajuato; 2002.

5. SAS. SAS/STAT User's Guide (Release 8.2, 6th Ed.). Cary NC: SAS. Inst. Inc., 2001.

6. Peña $F$, Doménech $V$, Acero $R$, Perea $J$, García A. Efecto de sistemas de crianza (leche de cabra vs. sustitutivo lácteo) y sexo en cabritos de raza Florida sobre su crecimiento y características de la canal. Rev Cient (FCV-LUZ) 2009; 19: 619-629.

7. Martínez JC, Rivera JP, González A. Preweaning performance of crossbred goat kids, from Juventino Rosas, Guanajuato. Proceeding of the 9th International Conference on Goats; 2008 Ago 31-Sep 5; Querétaro, Querétaro, México. International Goat Association; 2008.

8. Merlos MI, Martínez RD, Torres G, Mastache AA, Gallegos J. Evaluación de características productivas en cabritos Boer x local, Nubia $x$ local y locales en el trópico seco de Guerrero, México. Vet Méx 2008; 39: 323333.

9. Leal MJ, Gonçalves HC, Silva M, Rodrigues L, Lara GI, Lemos BB, Giassetti AP. Desempenho e medidas biométricas de caprinos de diferentes grupos raciais. Rev Bras Zootec 2007; 36: 635-642.
10. Mellado M, Mellado J, Garcia JE, Lopez R. Lifetime reproductive performance of goats as a function of growth traits and reproductive performance early in life. J Appl Anim Res 2005; 27: 113-116.

11. Martínez JC, Zúñiga JH, Soto JJ, de León R. Peso al nacer y a la venta de cabritos en un sistema de pastoreo trashumante en Burgos, Tamaulipas. Memoria. II Taller sobre sistemas de producción ovina del Noreste y Golfo de México; 2002 Nov 26 a 29; Cd. Victoria, Tamaulipas, México. Universidad Autónoma de Tamaulipas; 2002.

12. Martínez JC, Rivera JP, Lucero FA, Castillo SP. Peso al nacer y al destete de cabritos de razas lecheras en la zona del Bajío. Memoria del XXXIV Congreso Nacional de Buiatría; 2010 Ago 5 a 7; Monterrey, Nuevo León, México. AMMVEB; 2010.

13. Sánchez C, García M. Comparación de características productivas en caprinos con suplementación de bloques multinutricionales. Zoot Trop 2001; 19: 393-405.

14. Meneses R, Pérez P, Pittet J, Galleguillos P, Morales MS. Estrategia de alimentación durante la crianza de crías caprinas criollas. Agric Téc 2001; 61: 171-179.

15. Hernández ZJS, Herrera GM, Rodero SE, Vargas LS, Villarreal EO, Reséndiz MR, Carreón LL, Sierra VAC. Tendencia en el crecimiento de cabritos criollos en sistemas extensivos. Arch Zootec 2005; 54: 429-436.

16. Díaz GM, Ochoa MA, Torres G, Bisset P, Urrutia J, Morón FJ. Efectos de la inclusión de un concentrado de proteína de soya y dos fuentes de energía en el substituto de leche en el comportamiento productivo de cabritos nubios. Rev Cient (FCV-LUZ) 2007; 17: 597-605. 